\section{Commentary}

\section{A RICHENS}

Department of Pharmacology and Materia Medica, Welsh National School of Medicine, Cardiff.

Rylance and Moreland draw attention to the advantages of monitoring salivary anticonvulsant drug levels rather than blood levels in children. The non-invasive nature of this investigation, together with the fact that the salivary level reflects the free and pharmacologically-active concentration in plasma rather than the total concentration, make this method attractive. However, three difficulties arise which need stressing. Firstly, the measurement should be confined to phenytoin and carbamazepine because the salivary concentrations of the other antiepileptic drugs do not accurately reflect the free concentration. Secondly, the problem of oral contamination with liquid and chewable preparations is a real one, as Rylance and Moreland point out, and care has to be taken to ensure that the sampling is at least 5 hours after dosing. Thirdly, the concentration of the drugs in saliva is much lower than in plasma (one-tenth of the plasma concentration for phenytoin and one-quarter for carbamazepine). A substantial proportion of the levels encountered will therefore be around the limit of sensitivity of routine analytical techniques and the theoretical advantage of salivary levels may be more than offset by inaccuracy of measurement. Experience has shown ${ }^{1}$ that the coefficient of variation of analyses increases with decreasing concentration and may become of such a magnitude that the result may become more misleading than helpful. Although it is possible to achieve greater accuracy (as Rylance and Moreland have done), standards in most routine laboratories currently fall short of those necessary for salivary sampling to be a satisfactory substitute for venepuncture.

\section{Reference}

${ }^{1}$ Griffiths A, Hebdige S, Perucca E, Richens A. Quality control in drug measurement. Therapeutic Drug Monitoring $1980 ; 2$ : 51-9.

\title{
Early infantile variant of Krabbe globoid cell leucodystrophy with lung involvement
}

\author{
J $T$ R CLARKE, R L OZERE, AND V W KRAUSE
}

Department of Paediatrics and Department of Pathology, Dalhousie University, Izaak Walton Killam Hospital for Children, and Atlantic Research Centre for Mental Retardation, Halifax, Nova Scotia, Canada

SUMMARY An 8-week-old boy presented with a history of irritability, progressive feeding difficulty, generalised weakness, tachypnoea, and minor motor seizures. The clinical course was characterised by rapidly progressive respiratory failure, and neurological deterioration culminating in death at age 15 weeks. Electron microscopical examination and histological studies of the lung showed the presence of numerous intra-alveolar and a few interstitial macrophages. Enzyme studies and subsequent histopathological studies on brain confirmed the diagnosis of an unusual variant of Krabbe globoid cell leucodystrophy.

Krabbe globoid cell leucodystrophy (GLD) is a hereditary degenerative brain disease caused by lack of the enzyme, galactosylceramide $\beta$-galactosidase. ${ }^{1}$ Typically it is characterised by the onset at between 4 and 8 months of progressive developmental retardation, failure to thrive, seizures, spasticity, and blindness, culminating in death by age $2-3$ years. We report here an unusual, early-infantile variant of GLD in which primary lung involvement was prominent.

\section{Case report}

The patient was a boy, the fourth child born to apparently unrelated parents. The antenatal history and birth had been normal. Birthweight was $4170 \mathrm{~g}$. From the first day of life, he showed pronounced irritability and twitchiness. By age 2-3 weeks he had progressive feeding difficulty and became increasingly inactive and drowsy. Weight gain ceased. Breathing became rapid and was punctuated by frequent sighing. Two weeks before admission, occasional brief right-sided seizures had been noted.

On admission to hospital at 8 weeks he weighed $4395 \mathrm{~g}$; his length was $57 \mathrm{~cm}$ and head circumference $38 \mathrm{~cm}$. He was afebrile, wasted, and drowsy, and 